2009, 113, 8222-8226

Published on Web 05/27/2009

\title{
Excess Electron Solvation in an Imidazolium-Based Room-Temperature Ionic Liquid Revealed by Ab Initio Molecular Dynamics Simulations
}

\author{
Zhiping Wang, ${ }^{\dagger}$ Liang Zhang, ${ }^{\dagger}$ Xiaohua Chen, ${ }^{\dagger}$ Robert I. Cukier,${ }^{\ddagger}$ and Yuxiang Bu${ }^{*, \dagger}$ \\ The Center for Modeling \& Simulation Chemistry, Institute of Theoretical Chemistry, Shandong University, \\ Jinan, 250100, People's Republic of China, and Department of Chemistry, Michigan State University, \\ East Lansing, Michigan 48823
}

Received: March 21, 2009; Revised Manuscript Received: May 12, 2009

\begin{abstract}
We present the first approach to the excess electron solvation in a novel medium, room-temperature ionic liquid, using ab initio molecular dynamics simulation techniques in this work. Results indicate that an excess electron can be solvated in the $[\mathrm{dmim}]^{+} \mathrm{Cl}^{-} \mathrm{IL}$ as long-lived delocalized states and two short-lifetime localized states, one a single-cation-residence parasitical type and the other a double-cation-based solvated type state. The presence of a low-lying $\pi^{*}$-LUMO as the site of excess electron residence in the cation moiety disables the $\mathrm{C}-\mathrm{H}$ unit as a $\mathrm{H}$-bond donor, while the aromaticity requirement of the rings and the effect of the counterion $\mathrm{Cl}^{-}$'s make the resulting ion pairs a weak stabilizer for an excess electron. Although no large solvent reorganization in IL was found at the picosecond scale, the IL fluctuations sufficiently modify the relative energy levels of the excess electron states to permit facile state-to-state conversion and adiabatic migration. The binding energy of the excess electron is only $\sim 0.2 \mathrm{eV}$, further indicating that it is in a quasi-free state, with a large drift mobility, suggesting that ILs are unreactive and promising mediators for transport of excess electrons, in agreement with the experimental findings. The present study provides insight into the novel electron solvation character in a new class of promising media for physical and chemical processes, which are fundamental for understanding of electron migration mechanisms in IL-based applications.
\end{abstract}

The nature of an excess electron (EE) in various media has been extensively explored because of its fundamental importance in Chemistry and Physics and its relevance to a large class of physical and chemical phenomena associated with charge migration, radical reactions, and polarons. ${ }^{1,2}$ Although the EE has been shown to exist in either solvated and/or surface states in some molecular solvents ${ }^{2-6}$ and in a localized F-center-like state in alkali halide molten salts, ${ }^{7,8}$ its properties and transport mechanisms in other media remain poorly understood. In particular, recently developed room-temperature ionic liquids (IL) appear to be promising "green" media and have found many intriguing applications in synthetic chemistry, separation science, materials, and electrochemical devices such as batteries and solar cells. ${ }^{9}$ However, the states and evolution dynamics of migrating electrons in IL are still relatively unexplored.

IL are also an interesting topic for basic science, and their characteristic properties are being investigated with various techniques. ${ }^{10}$ ILs are a special ionic "molten salt" characterized as a liquid at or near room temperature, and they may have novel solvation properties. Their ionic nature implies that they might be able to affect efficient charge separation and to modulate charge migration via a solvent-mediated pathway, as demonstrated by a few recent experimental studies. ${ }^{10}$ They are, therefore, much different from conventional polar molecular solvents, with favorable stabilization and transport roles for an

\footnotetext{
* To whom correspondence should be addressed. E-mail: byx @sdu.edu.cn.

${ }^{\dagger}$ Shandong University.

* Michigan State University.
}

10.1021/jp902575s CCC: $\$ 40.75$
EE. For example, scavenging of so-called "dry" (not fully solvated) electrons by added solutes can occur on much faster time scales than that required by bulk reorganization of the solvent. ${ }^{10}$ In addition, an EE in IL exhibits a wide variety of electric and magnetic properties due to the dramatic competition between the long-range Coulomb attractions and short-range repulsion effects. Hence, a molecular-level understanding of the solvation and transport properties of an EE in this novel solvent medium is of great interest.

Here, we present ab initio molecular dynamic (AIMD) simulations and density functional theory (DFT) calculations of the structural character and evolution dynamics of an EE in IL. Our aim is to clarify the nature of the states of an EE in IL using as the IL the 1,3-dimethylimidazolium chloride ([dmim $]^{+} \mathrm{Cl}^{-}$, Figure 1) system.

An EE trapped in IL $(\mathbf{e} \cdots \mathrm{IL})$ was simulated in a periodically repeated cubic cell (cell parameter of $11.62 \AA$ ) consisting of eight ionic pairs plus one $\mathrm{EE}$, corresponding to the experimental density of $1.123 \mathrm{~g} / \mathrm{cm}^{3}$. Simulations were carried out within the canonical NVT ensemble with the system temperature kept at around $425 \mathrm{~K}$ by use of a Nosé-Hoover chain of thermostats. A 1 fs time step was used to ensure good control of the conserved quantities. The AIMD simulations used the nonlocal BLYP gradient corrected functional and DNP basis set. ${ }^{11}$ They were started from a configuration of the neutral IL system, and the simulation procedure may be described as follows. First, the IL were equilibrated with a classical MD simulation for 2 ns, followed by further AIMD simulation for $5 \mathrm{ps}$. Then, the following AIMD trajectory data were recorded for 8 ps for 


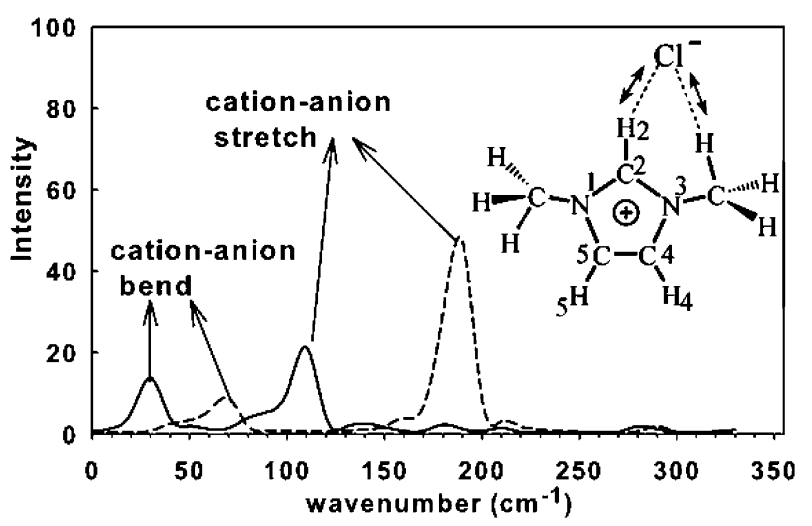

Figure 1. Ab initio calculated far-infrared vibrational spectrum of the $[\mathrm{dmim}]^{+} \mathrm{Cl}^{-}$(dashed curve) and $\mathbf{e} \cdots[\mathrm{dmim}]^{+} \mathrm{Cl}^{-}$(solid curve) ionic pairs. Both have two main peaks in the low-frequency range, as denoted by the arrows, which can be assigned to cation-anion bending and stretching modes.
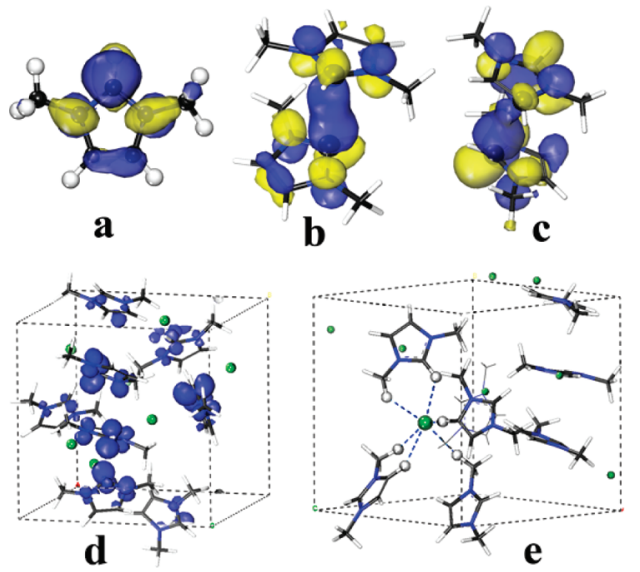

Figure 2. (a) (Parasitical state), (b), and (c) (solvated states) denote three localized states of the EE in $[\mathrm{dmim}]^{+} \mathrm{Cl}^{-} \mathrm{IL}$, displayed by SOMO. (d) The spin density isosurface plot corresponding to the delocalized state (contoured at 0.04). (e) A representative snapshot for the neutral IL containing possible H-bonds and the pseudoparallel configuration.

statistical analysis. Further, for e $\cdots$ IL, the AIMD simulation was started from the above AIMD-equilibrated configuration with the addition of an EE. After equilibration for $\sim 5 \mathrm{ps}$, the following 7 ps AIMD trajectory was recorded for analysis. In addition, another AIMD e... IL trajectory was generated in a parallel simulation by starting from the end of an independent classical MD run.

First, the microstructure of IL was analyzed in terms of suitable radial distribution functions. A considerable local nearorder was observed for the IL structure. The first solvation shell of $[\mathrm{dmim}]^{+}$is characterized by a set of $\mathrm{Cl}^{-}$ions localized in the neighborhood of the ring H's. The contacts are quite directional due to the dipole moment associated with the $\mathrm{C}-\mathrm{H}$ bonds, suggesting that the favored binding sites for $\mathrm{Cl}^{-}$are $\mathrm{H}(2,4,5)$, with a preference for $\mathrm{H}(2)^{12}$ (Figure 1). The number of $\mathrm{C}-\mathrm{H} \cdots \mathrm{Cl}$ contacts was monitored during the AIMD run using a purely geometrical selection criterion $\left(\mathrm{r}_{\mathrm{H}} \cdots \mathrm{Cl}<3.0 \AA\right)$ and was found to be up to 6 , indicating a favorable H-bonding network that, in addition to the dominant Coulombic stabilizing force, governs the IL structure. Nearest-neighbor cations with an average distance of $4 \AA$ were oriented such that their ring planes were approximately parallel to each other (Figure 2e). These simulation results for IL agree well with those from experiments and other theoretical methods, ${ }^{13,14}$ indicating that

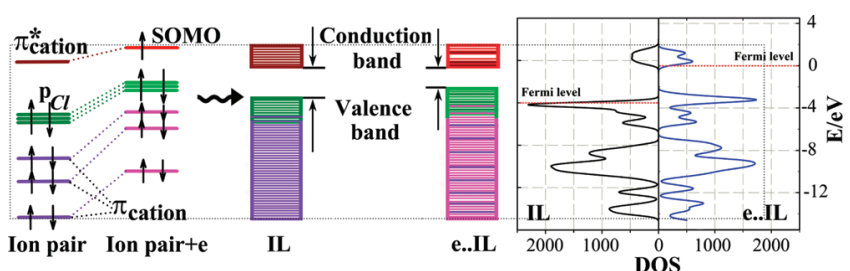

Figure 3. Correlation diagram of the density of states (DOS) plots of e...IL (blue) and IL (black) with the band structures and main orbitals of the ion pairs. Compared with IL, the addition of an EE leads to valence band up-shifting and thus destabilization of the IL system. At the top of the valence band, a weak peak is split out from a strong peak $\left(\mathrm{Cl}^{-}\right.$doubly occupied p orbital set, green) due to EE attachment, which originates from the nearly degenerate HOMO and HOMO-1 (both $\pi$-type) of each cation ring. At the same time, the conduction band is also split into two peaks, contributed to by the nearly degenerate LUMO and LUMO +1 (both $\pi^{*}$-type) of each cation ring.

the system size used here is adequate. It is noteworthy that the $[\mathrm{dmim}]^{+} \mathrm{Cl}^{-}$ion pair lowest unoccupied molecular orbital (LUMO) actually is a cation $\pi^{*}$-type LUMO, and thus, the ion pair is a delocalized $\pi^{*}$-type electron hole, and electron acceptance is not favorable due to the aromatic requirement of imidazolium. In addition, the LUMOs of all of the ion pairs make up a "LUMO" set for IL, and their denser population leads to a conduction band with a gap of $2.7 \mathrm{eV}$ from the valence band (Figure 3). This character is very important because it governs the survival state and transport property of an EE in IL.

Note that since simulation times of nanoeconds are needed to equilibrate ionic solutions, the effect of ion diffusion on EE transport cannot be obtained at present by AIMD simulations. Thus, on the AIMD simulation time scale, no significant ion diffusion in IL was observed, and the same is true in the subsequent AIMD simulations of $\mathbf{e}^{\cdot \cdot \cdot I L}$. However, fluctuation of the near-order IL structure can sufficiently affect the dynamics of the EE, and as we find, picosecond scale dynamics make significant contributions to the overall relaxation of $\mathbf{e} \cdots$ IL. In contrast with normal solutes, the EE could and does prefer to occupy the $\pi^{*}$-type hole over the cation rather than produce a reorganized cavity, the latter found for an EE in water. The dominant Coulombic and favorable $\mathrm{H}$-bond interactions between cations and anions effectively prevent the cations from reorganizing away from their mobile "cage" of anions. Thus, it is understandable that no solvation cavity for EE was observed in the AIMD simulation, though on a much longer time scale, there can be effects from ionic diffusion. Compared with the neutral simulation results, although the favored binding sites for $\mathrm{Cl}^{-}$ ions are the ring H's $(\mathrm{H}(2,4 / 5))$, the same is not true for the EE. It prefers to occupy the cation $\pi^{*}$-type LUMO rather than be $\mathrm{C}-\mathrm{H}$-dipole-bound, revealing a distinct difference in the preference of the IL cations for these two kinds of single negative charges $\left(\mathrm{Cl}^{-}\right.$versus $\left.\mathrm{e}^{-}\right)$, as confirmed by similar results obtained using both MP2 and DFT methods for the isolated $[\mathrm{dmim}]^{+}, \mathbf{e} \cdots[\mathrm{dmim}]^{+}, \mathbf{e} \cdots[\mathrm{dmim}]^{+} \mathrm{Cl}^{-}$, and $\mathbf{e} \cdots$ $\left([\mathrm{dmim}]^{+} \mathrm{Cl}^{-}\right)_{2}$ structures. These results also indicate that the inclusion of dispersion effects is not crucial in determining the structure and electronic state. These observations are consistent with the experimental and theoretical reports indicating that imidazolium-based IL are poor H-bond donors ${ }^{15}$ and that their $\mathrm{C}-\mathrm{H}$ groups cannot effectively bind an EE.

Despite the lack of significant bulk reorganization for $\mathbf{e} \cdot \cdots \mathrm{IL}$ partly due to the limited time scale of the present simulation, a marked variation was observed by monitoring the selected $\mathrm{H} \cdot \mathrm{Cl}^{-}$contacts, which is presumably associated with the local 

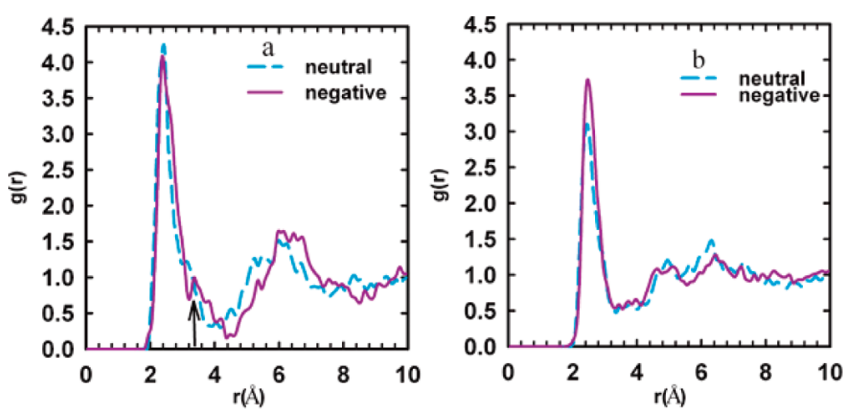

Figure 4. Site-site radial distribution functions $g(r)$. (a) The $g(r)$ between $\mathrm{H}(2)$ atoms and $\mathrm{Cl}^{-}$for IL (cyan) and $\mathbf{e} \cdots \mathrm{IL}$ (purple) systems, respectively. (b) The $g(r)$ between $\mathrm{H}(4 / 5)$ atoms and $\mathrm{Cl}^{-}$for two systems. Appearance of a weak peak pointed to by a black arrow and a slight shift to large $r$ of the secondary peak indicate the effect of the $\mathrm{EE}$ on the $\mathrm{H}(2) \cdots \mathrm{Cl}^{-} \mathrm{H}$-bond.

structure of the ion pair. That is, EE attachment leads to a considerable elongation of the local $\mathrm{H}(2) \cdots \mathrm{Cl}^{-} \mathrm{H}$-bonds, as shown in Figure 4a. In addition to the pronounced peak of $g(r)$ associated with $\mathrm{H}(2)$ and $\mathrm{Cl}^{-}$at $r \approx 2.3 \AA$ for both IL and e...IL, which accounts for the normal $\mathrm{H}(2) \cdots \mathrm{Cl}^{-}$contact distribution in the neat IL, ${ }^{14}$ a shoulder peak appears at $r \approx 3.3$ $\AA$ in $g(r)$ for $\mathbf{e} \cdots$ IL. Clearly, this new weak peak originates from the EE effect on the $\mathrm{H}(2) \cdots \mathrm{Cl}^{-}$interaction. The observed distance distributions are also supported by the calculated $\mathrm{H}(2) \cdots \mathrm{Cl}^{-}$distances for the neutral and negative ionic clusters $\left(\left([\mathrm{dmim}]^{+} \mathrm{Cl}^{-}\right)_{2}, 2.1 \AA\right.$, versus $\left.\left(\mathbf{e} \cdots\left([\mathrm{dmim}]^{+} \mathrm{Cl}^{-}\right)_{2}\right), 3.2 \AA\right) .{ }^{16}$ This observation indicates that $\mathrm{EE}$ attachment considerably weakens the $\mathrm{H}(2) \cdots \mathrm{Cl}^{-} \mathrm{H}$-bond. In contrast, it does not induce significant changes for the $\mathrm{H}(4 / 5) \cdots \mathrm{Cl}^{-}$interaction, as demonstrated by $g(r)$ (Figure 4 b) and the calculated $\mathrm{H}(4 / 5) \cdots \mathrm{Cl}^{-}$ contact distances in both systems. These observations for the contact distributions reveal that the attached EE mainly resides at the $\mathrm{N}(1)-\mathrm{C}(2)-\mathrm{N}(3)$ regions of the cations, as confirmed by the significant red shifts of the $\mathrm{H}(2) \cdots \mathrm{Cl}^{-}$stretching peak (by $\sim 80 \mathrm{~cm}^{-1}$ ) (Figure 1) and the electronic adsorption peak (by $\sim 2.6 \mathrm{eV}$ ) for the $[\mathrm{dmim}]^{+} \mathrm{Cl}^{-}$and $\mathbf{e} \cdots[\mathrm{dmim}]^{+} \mathrm{Cl}^{-}$ion pairs. These are in agreement with the results of the NAO charge analysis shown in Table S2c, Supporting Information.

The electronic state corresponding to the $\mathrm{EE}$ in IL is characterized by the alternating appearance of localized and delocalized $\pi$-type electron cloud distributions, unambiguously being neither a dipole-bound nor a reduced state of a cation. Inspection of the singly occupied molecular orbitals (SOMO) of the snapshots in the AIMD simulation for $\mathbf{e} \cdot \cdot \mathrm{IL}$ reveals that, for both cases, $\pi^{*}$-type orbitals over the imidazolium rings (actually a $\pi *$-type "LUMO" set (or empty band) of IL) are the sites of residence of the EE, which is distinctly different from those (the end-cation $\sigma$-type orbital set) found for EEs in solutions and alkali halide molten salts. All of the possible localized states for an EE in IL may be classified into two types, (i) single, ring-localized states (parasitical state, ${ }^{17}$ Figure $2 \mathrm{a}$ ) in which the EE occupies the IL LUMO originating from the $\operatorname{LUMO}\left(\pi^{*}\right)$ of a single imidazolium ring and (ii) double, ring-localized states (solvated state) in which the EE occupies the IL LUMO formed by linearly combining $\pi^{*}$-type LUMOs of two proximal imidazolium rings via a $\mathrm{C}(2) \cdots \mathrm{C}(2)$ contact faceto-face overlap (Figure 2 b) or via a $C(2) \cdots C(4 / 5)$ contact overlap (Figure 2c). In contrast, for the delocalized state (Figure 2d), the EE occupies the IL LUMO formed by a linear combination of a set of cation LUMOs. Clearly, all of the above states differ in an essential manner from the dipole- bound, solvated, and bonded states found for EEs in solutions or solids. The distinct appearance of these states can be understood from the LUMO nature of the cations. Because of the bulk fluctuations of IL, the arrangement and components of the orbitals in the empty LUMO band vary in an irregular fashion along the $\mathbf{e} \cdot$ - IL trajectory. At a certain moment, the LUMO of a particular cation dominantly contributes to the IL LUMO, the bottom of the empty band, and thus, the EE localizes on this cation fragment, forming a parasitical state. As the evolution proceeds, the ion pair fluctuations lead to a slight reorganization of the IL structure. As a consequence, if two proximal cations (LUMOs) almost contribute to the IL LUMO, acceptance of the EE generates a solvated state. On the other hand, if more cations contribute to the IL LUMO, the EE exhibits a delocalized state. In general, if no additional electric field is present, IL fluctuations can drive the EE evolution from one state to another by changing the $\mathrm{Cl}^{-}$-mediated cation $\cdots$ cation interaction.

By monitoring the trajectory, we found that an EE in [dmim $]^{+} \mathrm{Cl}^{-}$IL is dominantly in the delocalized state because either of the two kinds of well-localized states only survive for $10-20 \mathrm{fs},{ }^{17}$ which is markedly shorter than the survival time for the hydrated electron $(50-90 \mathrm{fs}){ }^{3}$ In contrast, conversion from one localized state to another takes 100-200 fs, or longer, which corresponds to the lifetime of the delocalized state. This observation may be attributed to four features. (a) The aromatic character of the ring weakens the binding ability of the cation in IL and thus diminishes its ability to accumulate an EE via its $\pi^{*}$-LUMO, with an electron affinity of only $0.4 \mathrm{eV}$. (b) The denser LUMO population of all cations in IL favors energetic redistribution of the IL $\pi^{*}$-LUMO empty band. (c) The pseudoparallel configuration of neighboring cation rings and the $\mathrm{Cl}^{-}$mediating role can provide a favorable pathway for rapid delocalization of the EE. (d) Localization, delocalization, or migration of an EE does not lead to significant structural reorganization of the cation fragments or the bulk IL. Overall, the EE in IL prefers the delocalized state to either of the localized states. Its state-to-state conversion rate is fluctuation-controlled, thus being more mobile than that in aqueous solution. ${ }^{3}$ The experimentally observed rate acceleration of electron transfer in $\mathrm{IL},{ }^{10}$ relative to comparable processes that are diffusion-controlled, is compatible with the above analysis of the electronic state evolution of $\mathbf{e} \cdot \cdot \mathrm{IL}$. Figure 5 depicts the time evolution of the EE Mulliken population over the eight cations, clearly reflecting the lifetimes of the EE states and the state-to-state conversion period.

The parasitical property of the EE in IL may be also verified by its energetics. For example, for the parasitical state EE, the energetic comparison between $\mathbf{e} \cdot \cdots$ IL and IL at the same configuration reveals that the vertical detachment energy of $\mathbf{e} \cdot$. IL is only $0.2 \mathrm{eV}$ (averaged over some snapshots in the period of this state), smaller than that $(0.75-1.92 \mathrm{eV})$ of the hydrated electron ${ }^{18}$ but quite close to the vertical attachment energy $(0.38 \mathrm{eV})$ of the $[\mathrm{dmim}]^{+} \mathrm{Cl}^{-}$ pair. Similarly small vertical detachment energies for other states are also observed. This further validates the AIMDbased conclusion that the EE in IL is weakly bound to the solvent components. All of these observations can be attributed to the EE-induced aromaticity variation of the imidazolium ring in IL cations. The attachment of an EE can destabilize the imidazolium ring, leading to considerable pyramidalization at its $\mathrm{N}$ sites and $\mathrm{C}(2)$-site. The aromaticity requirement of the ring and the IL electrostatic interactions 


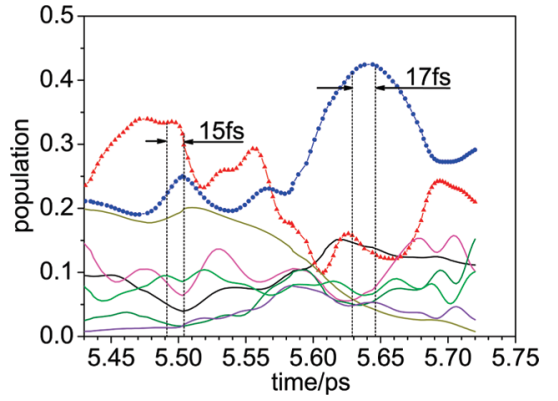

Figure 5. Population of the EE on eight cations in $\mathbf{e} \cdot \cdot \mathrm{IL}$ along the evolution time, as shown by eight curves with different colors. Two highlighted curves with solid red triangles and blue dots indicate the lifetimes of the localized and delocalized states and the EE transfer from one cation to another, or from one localized state to another via the intermediate delocalized state. The peak (at $\sim 5500$ fs) of the red curve corresponds to a solvated state, while the peak (at $\sim 5630 \mathrm{fs}$ ) of the blue curve corresponds to a parasitical state (single-cation-localized state). The alternating appearance of two peaks implies the transfer of an EE via a delocalized state over the period from $\sim 5510$ to $\sim 5630$ fs. The time record starts upon EE injection to the AIMD-equilibrated IL.

and $\mathrm{H}$-bond network of its cations and anions make the diffusion-based IL structural reorganization and cation distortion energetically unfavorable. These features lead to the parasitical character and high mobility behavior of the EE in IL.

Additionally, further orbital analysis along the AIMD trajectory shows that a $\pi^{*}$-orbital set that is densely populated and forms an empty band actually originates from the $\pi^{*}$ LUMOs of all of the cation moieties of the IL, with a very small bandwidth of less than $2.3 \mathrm{eV}$. Attachment of an EE significantly reduces the HOMO-LUMO gap, as is manifested in the density of states (DOS) plot (Figure 3), by upshifting the valence band. Compared with IL, in $\mathbf{e} \cdots \mathrm{IL}$, a weak peak is revealed that is split off from the strongest peak at the top of the valence band (the occupied $\mathrm{Cl}^{-}$p-orbital peak). The EE also down-shifts the IL LUMO (becoming a SOMO in $\mathbf{e} \cdot \mathrm{IL}$ ), thus leading to the above splitting and, in addition, a splitting of the empty band.

In summary, an EE can be trapped in the $[\mathrm{dmim}]^{+} \mathrm{Cl}^{-} \mathrm{IL}$ as two short-lifetime localized states, one a single-cationresidence parasitical type and the other a double-cation-based solvated type state. There also exist long-lived delocalized states. The presence of a low-lying $\pi^{*}$-LUMO as the site of $\mathrm{EE}$ residence in the cation moiety disables the $\mathrm{C}-\mathrm{H}$ unit as a $\mathrm{H}$-bond donor, while the aromaticity requirement of the rings and the effect of the counterion $\mathrm{Cl}^{-}$'s make the resulting ion pairs a weak stabilizer for an EE. Although no large solvent reorganization in IL was found at the picosecond scale, the IL fluctuations sufficiently modify the relative energy levels of the EE states to permit facile state-to-state conversion and EE adiabatic migration. The binding energy of the EE is only $\sim 0.2 \mathrm{eV}$, further indicating that it is in a quasi-free state with large drift mobility, suggesting that ILs are unreactive and promising mediators for transport of EEs, in agreement with the experimental findings. The present study provides insight into the novel electron solvation character in a new class of promising media for physical and chemical processes, which are fundamental for understanding of electron migration mechanisms in IL-based applications.

Acknowledgment. This work was supported by NSFC (20633060, 20573063), NCET, and Virt Lab for Comput Chem
\& SuperComputer Center of CNIC-CAS, MCBILIN at MSU, and High-performance Comput \& Simulation Platform at SDU Chem School.

Supporting Information Available: Details for molecular dynamics simulations; geometric details; relevant energetics; spin density and molecular orbital contours; radial distribution functions; electronic adsorption spectra; and state evolution of excess electron. This material is available free of charge via the Internet at http://pubs.acs.org.

\section{References and Notes}

(1) (a) Hart, E. J.; Boag, J. W. J. Am. Chem. Soc. 1962, 84, 4090. (b) Keene, J. P. Nature 1963, 197, 47.

(2) (a) Kevan, L. Acc. Chem. Res. 1981, 14, 138. (b) Jordan, K. D. Science 2004, 306, 618. (c) Verlet, J. R. R.; Bragg, A. E.; Kammrath, A.; Cheshnovsky, O.; Neumark, D. M. Science 2005, 307, 93. (d) Bragg, A. E.; Verlet, J. R. R.; Kammrath, A.; Cheshnovsky, O.; Neumark, D. M. Science 2004, 306, 669. (e) Paik, D. H.; Lee, I.; Yang, D.; Baskin, J. S.; Zewail, A. H. Science 2004, 306, 672. (f) Hammer, N. I.; Shin, J.; Headrick, J. M.; Diken, E. G.; Roscioli, J. R.; Weddle, G. H.; Johnson, M. A. Science 2004, 306, 675. (g) Turim, L.; Sheu, W.; Rossky, P. J. Science 2005, 309, 914. (h) Herbert, J. M.; Head-Gordon, M. Proc. Natl. Acad. Sci. U.S.A. 2006, $103,14282$.

(3) (a) Boero, M.; Parrinello, M.; Terakura, K.; Ikeshoji, T.; Liew, C. C. Phys. Rev. Lett. 2003, 90, 226403. (b) Skurski, P.; Rak, J.; Simons, J.; Gutowski, M. J. Am. Chem. Soc. 2001, 123, 11073-11074. (c) Baletto, F.; Cavazzoni, C.; Scandolo, S. Phys. Rev. Lett. 2005, 95, 176801.

(4) (a) Sommerfeld, T.; Jordan, K. D. J. Am. Chem. Soc. 2006, 128, 5828. (b) Herbert, J. M.; Head-Gordon, M. J. Am. Chem. Soc. 2006, 128, 13932. (c) Jordan, K. D.; Wang, F. Annu. Rev. Phys. Chem. 2003, 54, 367. (d) Nordlund, N.; et al. Phys. Rev. Lett. 2007, 99, 217406. (e) Bragg, A. E.; Verlet, J. R. R.; Kammrath, A.; Cheshnovsky, O.; Neumark, D. M. J. Am. Chem. Soc. 2005, 127, 15283-15295.

(5) (a) Tauber, M. J.; Stuart, C. M.; Mathies, R. A. J. Am. Chem. Soc. 2004, 126, 3414. (b) Tauber, M. J.; Mathies, R. A. J. Am. Chem. Soc. 2003, 125, 1394-1402. (c) Zhao, J.; Li, B.; Onda, K.; Feng, M.; Petek, H. Chem. Rev. 2006, 106, 4402-4427. (d) Onda, K.; Li, B.; Zhao, J.; Jordan, K. D.; Yang, J.; Petek, H. Science 2005, 308, 1154.

(6) (a) Yan, S. H.; Bu, Y.; Cukier, R. I. J. Chem. Phys. 2006, 124, 124314. (b) Chandler, D.; Leung, K. Annu. Rev. Phys. Chem. 1994, 45, 557. (c) Zhu, J.; Cukier, R. I. J. Chem. Phys. 1993, 99, 5384. (d) Lee, S.; et al. Phys. Rev. Lett 1997, 79, 2038.

(7) (a) Selloni, A.; Carnevali, P.; Car, R.; Parrinello, M. Phys. Rev. Lett. 1987, 59, 823. (b) Leone, A. M.; Weatherly, S. C.; Williams, M. E.; Thorp, H. H.; Murray, R. W. J. Am. Chem. Soc. 2001, 123, 218-222.

(8) (a) Xia, P.; Bloomfield, L. A. Phys. Rev. Lett. 1993, 70, 1779. (b) Zhang, L.; Yan, S. H.; Cukier, R. I.; Bu, Y. J. Phys. Chem. B 2008, 112, 3767.

(9) (a) Dzyuba, S. V.; Bartsch, R. A. Angew Chem.Int. Ed 2003, 42, 148-150. (b) Rogers, R. D.,Seddon, K. R., Eds.; Ionic Liquids: Industrial Applications for Green Chemistry; American Chemical Society: Washington DC, 2002. (c) Welton, T. Chem. Rev. 1999, 99, 2071. (d) Macfarlane, D. R.; Forsyth, M.; Howlett, P. C.; Pringle, J. M.; Sun, J.; Annat, G.; Neil, W.; Izgorodina, E. I. Acc. Chem. Res. 2007, 40, 1165. (e) Fukumoto, K.; Ohno, H. Angew. Chem. Int. Ed 2007, 46, 1852-1855. (f) Dupont, J.; Spencer, J. Angew. Chem. Int. Ed 2004, 43, 5296-5297.

(10) (a) Fumino, K.; Wulf, A.; Ludwig, R. Angew. Chem., Int. Ed. 2008, 47, 3830-3834. (b) Jones, C. B.; Haiges, R.; Schroer, T.; Christe, K. O. Angew Chem. Int. Ed 2006, 45, 4981-4984. (c) Vieira, R. C.; Falvey, D. E. J. Am. Chem. Soc. 2008, 130, 1552. (d) Shim, Y.; Jeong, D.; Manjari, S.; Choi, M. Y.; Kim, H. J. Acc. Chem. Res. 2007, 40, 1130. (e) Lockard, J. V.; Wasielewski, M. R. J. Phys. Chem. B 2007, 111, 11638. (f) Vieira, R. C.; Falvey, D. E. J. Phys. Chem. B 2007, 111, 5023. (g) Wishart, J. F.; Neta, P. J. Phys. Chem. B 2003, 107, 7261. (h) Katoh, R.; Yoshida, Y.; Katsumura, Y.; Takahashi, K. J. Phys. Chem. B 2007, 111, 4770. (I) Mele, A.; Roman, G.; Giannone, M.; Ragg, E.; Fronza, G.; Raos, G.; Marcon, V. Angew. Chem., Int. Ed. 2006, 45, 1123-1126. (j) Weingrtner, H. Angew. Chem. Int. Ed 2008, 47, 654-670.

(11) (a) All simulations were done with the $\mathrm{DMol}^{3}$ package, as implemented in Cerius 2, version 4.6; Accelrys Inc.: San Diego, CA, 2008. (b) Delley, B. J. Chem. Phys. 1990, 92, 508. (c) Delley, B. J. Chem. Phys. $2000,113,7756$.

(12) $\mathrm{A} \mathrm{Cl}^{-}$anion can interact with the $\pi$-manifold of the cation orbitals via a top site, forming a top conformer, but $\mathrm{Cl}^{-}$is very close to the $\mathrm{C}(2)-$ $\mathrm{H}(2)$ unit, and the top binding is slightly above the in-plane $\mathrm{H}(2)$ binding by $0.03 \mathrm{eV}$. Thus, we grouped it into the $\mathrm{H}(2)$ binding category.

(13) (a) Hunt, P. A.; Kirchner, B.; Welton, T. Chem.-Eur. J. 2006, 12, 6762. (b) Holbrey, J. D.; et al. Chem. Commun. 2003, 1636. 
(14) Bühl, M.; Chaumont, A.; Schurhammer, R.; Wipff, G. J. Phys. Chem. B 2005, 109, 18591.

(15) (a) Anderson, J. L.; et al. J. Am. Chem. Soc. 2002, 124, 14247. (b) The H-bond energies of $\left[\mathrm{dmim}^{+}\right] \mathrm{C}-\mathrm{H}(2,4 / 5) \cdots \mathrm{Cl}^{-}$are 1.71 and $1.39 \mathrm{eV}$, respectively, considerably smaller than that $(3.54 \mathrm{eV})$ of $\mathrm{HOH} \cdots \mathrm{Cl}^{-}$, calculated at the B3LYP/6-31+G* level of theory for isolated ion pairs.

(16) The calculated results were obtained at the B3LYP/6-31+G* level for the corresponding anionic and neutral clusters of the two ion pairs.

(17) In this case, an EE is localized on one cation, but the cation is not reduced. The cation actually plays the role of an attractor via its $\pi^{*}$-LUMO to bind an EE, and the EE may be viewed to reside parasitically on the cation(s). Therefore, we define it as a temporary parasitical state or a pseudoreduced state, which also differ from the dipole-bound state observed in EE hydration. Note that the lifetime for the two kinds of the localized states is defined as the residence time of the EE localized on one or two proximal imidazolium rings with $>50 \%$ population.

(18) (a) Coe, J. V.; et al. J. Chem. Phys. 1990, 92, 3980. (b) Coe, J. V. Int. Rev. Phys. Chem. 2001, $20,33$.

JP902575S 